\title{
A hyperspectral imaging system for mapping haemoglobin and cytochrome-c-oxidase concen- tration changes in the exposed cerebral cortex
}

\author{
Luca Giannoni, Frédéric Lange, Marija Sajic, Kenneth J. Smith, and Ilias Tachtsidis
}

\begin{abstract}
We present a novel hyperspectral imaging (HSI) system using visible and near-infrared (NIR) light on the exposed cerebral cortex of animals, to monitor and quantify in vivo changes in the oxygenation of haemoglobin and in cellular metabolism via measurement of the redox states of cytochrome-c-oxidase (CCO). The system, named hNIR, is based on spectral scanning illumination at 11 bands $(600,630,665,784,800,818,835,851,868,881$ and $894 \mathrm{~nm}$ ), using a supercontinuum laser coupled with a rotating Pellin-Broca prism. Image reconstruction is performed with the aid of a Monte Carlo framework for photon pathlength estimation and post-processing correction of partial volume effects. The system is validated on liquid optical phantoms mimicking brain tissue haemodynamics and metabolism, and finally applied in vivo on the exposed cortex of mice undergoing alternating oxygenation challenges. The results of the study demonstrate the capacity of hNIR to map and quantify the haemodynamic and metabolic states of the exposed cortex at microvascular levels. This represents (to the best of our knowledge) the first example of simultaneous mapping and quantification of cerebral haemoglobin and $\mathrm{CCO}$ in vivo using visible and NIR HSI, which can potentially become a powerful tool for better understanding brain physiology.
\end{abstract}

Index Terms-Hyperspectral imaging, wide-field optical imaging, near-infrared spectroscopy, brain metabolism, brain oxygenation, cytochrome-c-oxidase.

\section{INTRODUCTION}

$\mathrm{R}$ ECENT biomedical applications of hyperspectral imaging (HSI) have highlighted the potential of this optical modality to provide functional information on various in vivo physiological processes [1]-[3]. This is thanks to its multidimensional capacity of acquiring wide-field images at numerous contiguous wavelengths over a broad range of the electromagnetic spectrum (visible and infrared), thus combining spectral and spatial data with high resolution and sensitivity for biochemical molecules of interest [4]. In particular, in neuroimaging it has been demonstrated how HSI is able to map and quantify in vivo changes in the concentrations of oxyhaemoglobin $\left(\mathrm{HbO}_{2}\right)$ and deoxyhaemoglobin $(\mathrm{HHb})$ in the exposed cerebral cortex of both humans and animals, thus providing information about

Manuscript received September 30, 2020. This work was supported in part by the European Union's Horizon 2020 Research and Innovation Program under the Marie Sklodowska-Curie Grant 675332, in part by Wellcome Trust under Grant 104580/Z/14/Z, in part by Medical Research Council funding under Grant MR/S003134/1, and in part by Fondation Leducq, Rosetrees Trust, and UK Multiple Sclerosis Society. Corresponding author: Luca Giannoni,

L. Giannoni, F. Lange, and I. Tachtsidis are with the Department of Medical brain oxygenation and haemodynamics [5]. Hyperspectral brain imaging is often based on similar approaches and analyses typically used in near-infrared spectroscopy (NIRS) [6]: it involves measuring the absorption optical signatures of the two forms of haemoglobin, yet with much higher spatial resolution that is characteristic of wide-field optical imaging (WFOI) [5], [7], [8]. Furthermore, the use of multiple wavelengths provided by HSI (as to reconstruct complete spectra of each single pixel in the image), compared to the only 2-3 usually employed in most commercial NIRS instruments, has the additional advantage of extending the number of chromophores of interest that can be resolved. Indeed, complementarily to the haemodynamic and oxygenation information provided by cerebral haemoglobin, mapping and quantification of brain cellular metabolism could also be inferred using HSI by targeting the optical signature of the redox state of cytochrome-c-oxidase (CCO) [5].

$\mathrm{CCO}$ is the complex IV in the electron transport chain (ETC) of the mitochondria, and thus it is involved in more than $95 \%$ of the production of adenosine triphosphate (ATP) in cells. Its absorption properties vary between its oxidative (oxCCO) and reduced (redCCO) states, therefore by using its oxidised minus reduced differential extinction spectrum (diffCCO), it is possible to retrieve information about the redox changes in $\mathrm{CCO}$ and in brain tissue mitochondrial functions associated with changes in metabolism [9]. Since the extinction spectrum of diffCCO presents a predominant broad peak in the near-infrared (NIR) range $780-900 \mathrm{~nm}$, due to the copper $\mathrm{CuA}$ redox centres of $\mathrm{CCO}$ [10], a large number of wavelengths sampled in such interval is necessary in order to simultaneously and accurately resolve $\mathrm{HbO}_{2}, \mathrm{HHb}$ and oxCCO (as direct index of metabolic activity) [9]. This is currently done non-invasively via broadband NIRS (bNIRS), an optical spectroscopy technique that is based on white light illumination (at least 120 wavelengths) of the head through optic fibres and on collecting the reflected diffused NIR signal with a spectrometer [9], [11], [12]. However, such an approach is limited in terms of spatial resolution, as it typically samples large $\left(\mathrm{few} \mathrm{cm}^{3}\right)$ volumes of brain tissue, including extracerebral layers (scalp, skull and cerebrospinal fluid) [13].

Physics and Biomedical Engineering, University College London, London WC1E 6BT, UK (e-mail: 1.giannoni@ucl.ac.uk; f.lange@ucl.ac.uk; i.tachtsidis@ucl.ac.uk).

M. Sajic and K. J. Smith are with the Department of Neuroinflammation, UCL Queen Square Institute of Neurology, London, WC1N 3BG2, UK (email: m.sajic@ucl.ac.uk; k.smith@ucl.ac.uk). 
We previously demonstrated computationally the fitness of HSI in mapping and quantifying changes in the concentration of $\mathrm{HbO}_{2}, \mathrm{HHb}$ and oxCCO on the exposed cortex during hypoxia, using Monte Carlo (MC) simulations [14]. In the same MC studies we also proved that the use of only 8 optimallyselected NIR wavelengths in the range 780-900 $\mathrm{nm}(784,800$, $818,835,851,868,881$ and $894 \mathrm{~nm}$ ) provides minimal differences (less than 2\%) in the accuracy at simultaneously resolving haemoglobin and CCO signals, compared to the 'gold standard' of 121 wavelengths used in bNIRS [14]. We now present in this work the first in vivo application of HSI to image and monitor $\mathrm{HbO}_{2}, \mathrm{HHb}$ and oxCCO in the exposed cortex of mice, using the same 8 NIR spectral bands, plus the addition of 3 visible wavelengths $(600,630$ and $665 \mathrm{~nm})$. The combination of the visible and NIR wavelengths was selected to enhance image contrast from both haemoglobin and $\mathrm{CCO}$, while avoiding crosstalk from the optical signatures of other species of cytochrome below $600 \mathrm{~nm}$ [9]. In this paper we introduce a novel HSI benchtop system, named hNIR, and its validation in a controlled experimental setting, as well as its application to the exposed cerebral cortex of anesthetised mice during oxygenation challenges (hyperoxia, hypoxia and anoxia).

\section{MATERIAL AND METHODS}

\section{A. The hNIR system}

The hNIR system is based on spectral scanning HSI mode, illuminating the target sequentially at all the desired spectral bands and synchronously acquiring a single frame for each wavelength, in order to reconstruct a three-dimensional (3D) spatio-spectral dataset called hypercube (by stacking together all the spectral frames). A diagram of the overall configuration of the hNIR system is shown in Fig. 1.

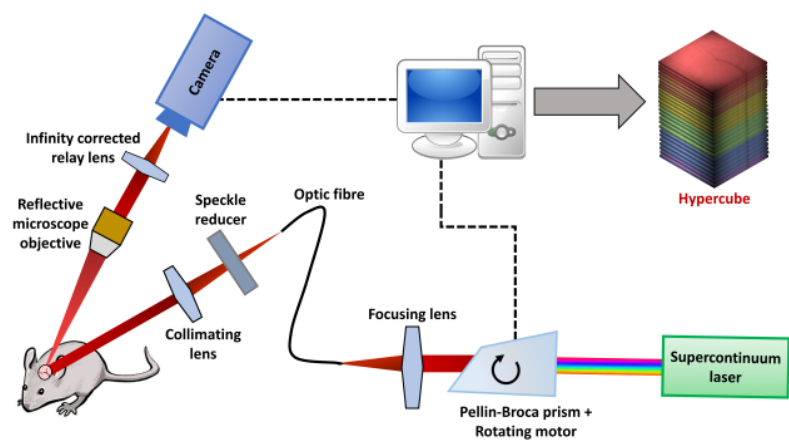

Fig. 1. Configuration of the hNIR setup, including spectral illumination side and imaging side. Broadband light emitted by the supercontinuum (SC) laser is dispersed in its wavelength constituents by the Pellin-Broca prism mounted on a rotating motor and then focused to a small-core optic fibre that only selects a narrow spectral band. At every rotation of the motor, a different band is scanned, which then illuminates the target. Reflected light form the target is then collected by a reflective objective, focused through a relay lens and recorded by a camera synchronised with the rotating motor.

The illumination side of hNIR is composed of a supercontinuum (SC) laser (Fianium WhiteLase SC440-40) that is coupled with a Pellin-Broca prism (Thorlabs ADB-10) mounted on a rotating motor (PI U-651.04) for sequential illumination of the target at 11 wavelength bands $(600,630,665,784,800,818$, $835,851,868,881$ and $894 \mathrm{~nm})$. The broadband output of the
SC laser is directed via optic fibre to the input side of the PellinBroca prism, which disperses the beam in its constituent spectral components. The dispersed beam reaches an achromatic lens (Thorlabs AC254-200-AB) with focal length of $200 \mathrm{~mm}$. This lens collimates the dispersed beam towards a small-core (10- $\mu \mathrm{m}$ diameter) optic fibre (Thorlabs M64L01), that is positioned at the focal point of the lens and it is used to illuminate the target. The optic fibre selects only a small portion of the spectrum of the dispersed beam (due to its small diameter). Rotating the motor (and thus the prism) in rapid sequence $(150 \mathrm{~ms}$ per band) allows the system to switch between all the spectral bands, each corresponding to a specific angular position of the motor. Finally, the narrow-band light that exits the optic fibre is directed towards the imaged target. Before illuminating it, the beam is filtered by a laser speckle reducer (Optotune LSR3005-6D-NIR), to mitigate the occurrence of speckle noise, and then further collimated using another achromatic doublet lens (Thorlabs AC254-030-AB) with focal distance of $30 \mathrm{~mm}$ (creating a final spot size on target of about $4 \mathrm{~mm}$ in diameter).

Image acquisition at each illuminating spectral band $(150 \mathrm{~ms}$ per frame) is performed via a scientific complementary metaloxide semiconductor (sCMOS) camera (Andor Zyla 5.5 USB 3 ), mounted on a 3D translation stage for correct placement. The camera is coupled to an optical assembly including: (1) a $15 \mathrm{x}$ reflective microscope objective (Thorlabs LMM-15XP01); and (2) an achromatic, infinity-corrected relay lens (Thorlabs TTL200-B). The optical assembly is used for light collection, focusing and magnification to the camera sensor, generating a field of view (FOV) of about $0.9 \times 1.1 \mathrm{~mm}$.

The hNIR system was characterised in terms of spectral, power and imaging performances. The complete characteristics and features of the hNIR system are reported in Table I.

TABLE I

TECHNICAL CHARACTERISATION OF HNIR

\begin{tabular}{cc}
\hline \hline Specifications: & Illumination side: \\
\hline Illumination mode: & Spectral scanning \\
Available spectral range: & $380-2500 \mathrm{~nm}$ \\
Number of spectral bands: & 11 (visible and NIR) \\
Spectral resolution (FWHM): & $\sim 6-8 \mathrm{~nm}$ (visible) \\
Average output power per & $\sim 8-11 \mathrm{~nm}$ (NIR) \\
spectral band: & $\sim 60-95 \mu \mathrm{W}$ (visible) \\
Average switching rate between & $\sim 85-110 \mu \mathrm{W}$ (NIR) \\
spectral bands: & $\sim 150 \mathrm{~ms} / \mathrm{band}$ \\
\hline Specifications: & Imaging side: \\
\hline Type of detector: & sCMOS \\
Spatial resolution: & $2560 \times 2160$ pixels \\
Pixel size: & $6.5 \mu \mathrm{m}$ \\
Frame rate: & 40 fps (at full format) \\
FOV: & $0.9 \times 1.1 \mathrm{~mm}$ \\
Sensitivity (QE): & $60 \%$ at $600 \mathrm{~nm}$ \\
Shutter mode: & Global \\
Integration time: & $150 \mathrm{~ms}$ \\
Hypercube rate: & 0.3 hypercubes/s \\
Maximum dynamic range: & $33,000: 1$ \\
\hline \hline
\end{tabular}

NIR, near-infrared; FWHM, full-width at half maximum; sCMOS: scientific complementary metal-oxide semiconductor; FOV, field of view; QE, quantum efficiency. 
In particular, spatial resolution of hNIR under broadband illumination and for each spectral band of the system was assessed using a positive resolution test target, the USAF 1951 (Thorlabs R1L1S1P). For both tests (broadband and single band illumination), the spatial resolution of hNIR was found equal to 228 $\mathrm{lp} / \mathrm{mm}$, corresponding to $2.19 \mu \mathrm{m}$. An example of the result using broadband illumination is shown in Fig. 2.
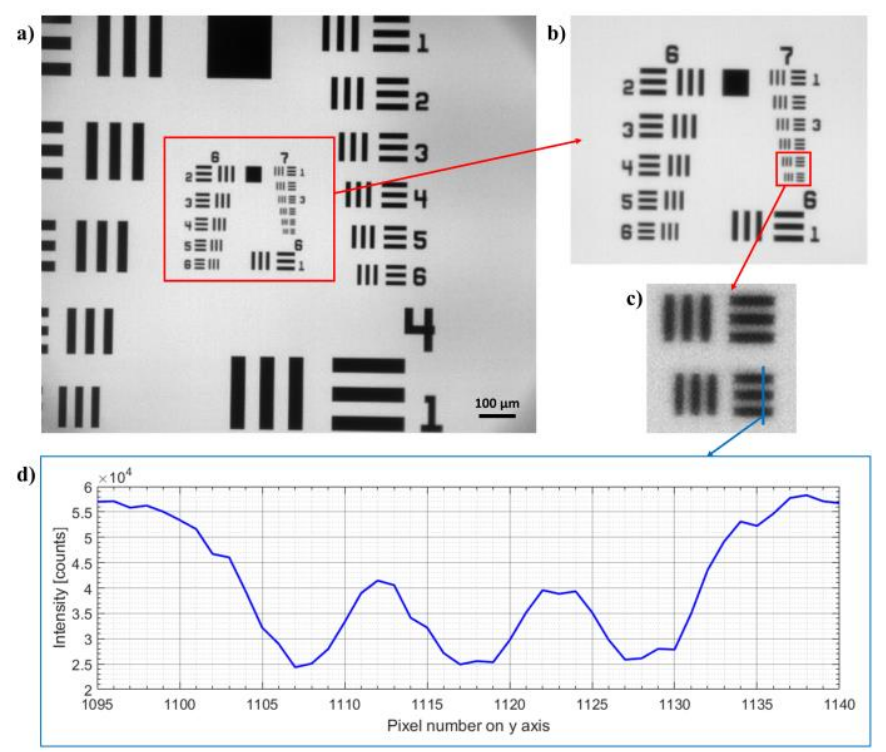

Fig. 2. a) Picture of a USAF 1951 resolution target obtained with the imaging side of hNIR using broadband illumination, where the smallest resolved groups (in red) are identified; b) Zoom on the smallest resolved group (Group 7), where the smallest resolved element is identified (in red); c) Zoom on the smallest resolved element (element 6); d) Line profile of the smallest resolved element (Group 7 Element 6).

\section{B. Data processing and analysis with $h N I R$}

After hyperspectral data collection, in the form of several hypercubes (for every experimental phase of the desired procedure) each composed of a number of frames equal to the number $M$ of selected spectral bands (up to 11 for hNIR), a data processing and analysis methodology is implemented and conducted on these raw hypercubes in order to reconstruct spatial maps of the relative changes in the concentrations of $\mathrm{HbO}_{2}$, $\mathrm{HHb}$ and oxCCO on the target FOV. The data processing procedure is summarised in a flowchart diagram in Fig. 3.

Firstly, the raw spectral images of all hypercubes acquired with hNIR are filtered to further reduce the influence of speckle noise generated by the laser source using a 2D-adaptive, lowpass Wiener filter [15], [16]. Afterwards, all the filtered hyperspectral cubes (for each experimental phase dataset) are corrected via subtraction of a dark reference hypercube acquired with the camera sensor completely covered, at the same integration time as the rest of the data. The last step in the pre-processing of the hyperspectral data involves time integration and averaging. In particular, the hypercubes of the baseline condition in the procedure are time-averaged over their whole acquisition time, as to obtained a single averaged baseline hypercube out of them, having intensity $I_{\text {baseline }}(\lambda)_{k, l}$ for every spectral band $\lambda$ and in each pixel $k, l$. Conversely, the other hypercubes (typically corresponding to a specific experimental phase) are time-

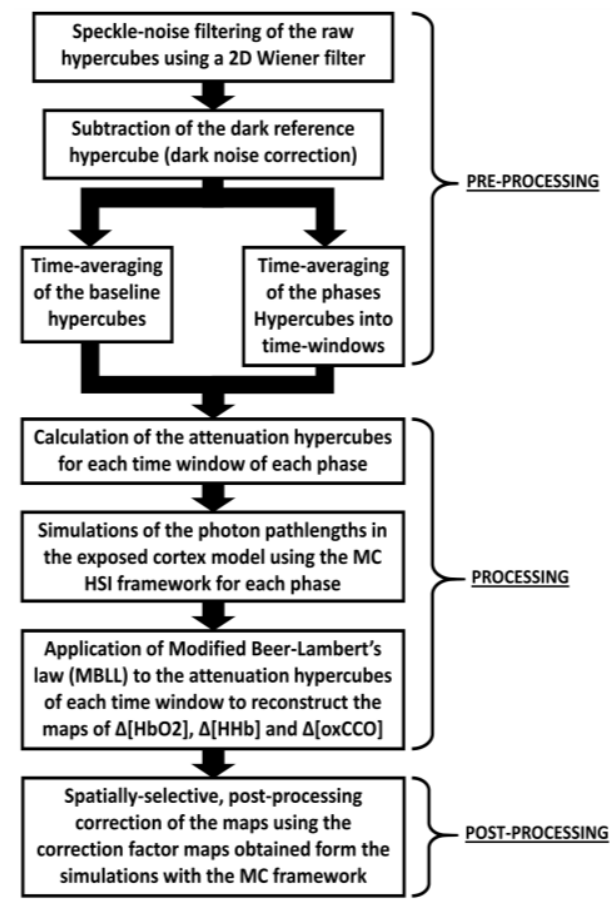

Fig. 3. Flowchart diagram describing step-by-step the data processing methodology applied to the hyperspectral data collected with hNIR

averaged into a number of specific time windows, according to the acquisition time of the given dataset. These averaged hypercube for each time window $\Delta t$ have intensity $I_{\Delta t}(\lambda)_{k, l}$ for every spectral band $\lambda$ and in each pixel $k, l$.

Following pre-processing, a modified Beer-Lambert's law (MBLL) approach [17] is applied on the time-averaged hypercubes of each phase as to reconstruct spatial maps of the relative changes $\Delta\left[\mathrm{HbO}_{2}\right], \Delta[\mathrm{HHb}]$ and $\Delta[\mathrm{oxCCO}]$ in the concentrations of $\mathrm{HbO}_{2}, \mathrm{HHb}$ and oxCCO of the imaged FOV. Firstly, attenuation between the baseline and a given time window $\Delta t$ of the $i$-th condition is calculated for each pixel $k, l$ and every selected spectral band $\lambda$ of the hypercubes:

$$
\Delta A(\lambda)_{k, l}=-\log _{10}\left(\frac{I_{\Delta t}(\lambda)_{k, l}}{I_{\text {baseline }}(\lambda)_{k, 1}}\right) .
$$

Afterwards, MBLL is applied pixel-wise (for each pixel $k, l$ ) on the attenuation values in (1) to calculate the corresponding haemodynamic and metabolic maps of $\Delta\left[\mathrm{HbO}_{2}\right], \Delta[\mathrm{HHb}]$ and $\Delta[\mathrm{oxCCO}]$, for each time window $\Delta t$ of all the experimental phases. Thus, for each pixel $k, l$ of the pre-processed hypercubes the following systems of algebraic equations are set:

$$
\left[\begin{array}{c}
\Delta A_{k, l}\left(\lambda_{1}\right) \\
\Delta A_{k, l}\left(\lambda_{2}\right) \\
\vdots \\
\Delta A_{k, l}\left(\lambda_{M}\right)
\end{array}\right]=\operatorname{diag}\left(P L_{k, l}\left(\lambda_{1}\right), \ldots, P L_{k, l}\left(\lambda_{\mathrm{M}}\right)\right) \times
$$

$$
\times\left[\begin{array}{ccc}
\varepsilon_{\mathrm{HbO}_{2}}\left(\lambda_{1}\right) & \varepsilon_{\mathrm{HHb}}\left(\lambda_{1}\right) & \varepsilon_{\mathrm{diffCCO}}\left(\lambda_{1}\right) \\
\varepsilon_{\mathrm{HbO}_{2}}\left(\lambda_{2}\right) & \varepsilon_{\mathrm{HHb}}\left(\lambda_{2}\right) & \varepsilon_{\mathrm{diffCCO}}\left(\lambda_{2}\right) \\
\vdots & \vdots & \vdots \\
\varepsilon_{\mathrm{HbO}_{2}}\left(\lambda_{M}\right) & \varepsilon_{\mathrm{HHb}}\left(\lambda_{M}\right) & \varepsilon_{\mathrm{diffCCO}}\left(\lambda_{M}\right)
\end{array}\right] \times\left[\begin{array}{c}
\Delta\left[\mathrm{HbO}_{2}\right]_{k, l} \\
\Delta[\mathrm{HHb}]_{k, l} \\
\Delta[\mathrm{oxCCO}]_{k, l}
\end{array}\right] .
$$


The reference spectra for the molar extinction coefficients $\varepsilon_{\mathrm{HbO}_{2}}$ and $\varepsilon_{\mathrm{HHb}}$ of haemoglobin are taken from Matcher et al [14], [18], while the oxidised-reduced differential molar extinction coefficients $\varepsilon_{\text {diffCCO }}$ for CCO are calculated from data measured in vivo at UCL by J. Moody [9], [14].

The maps (for each wavelength $\lambda$ ) of the spatial distributions of the average total pathlength $P L_{k, l}(\lambda)$ of the detected photons on the FOV in (2) are estimated by running MC simulations using the methodology we developed and presented in Giannoni et al [14]. A high-contrast picture of the FOV, that is obtained with the imaging side of the hNIR system using broadband illumination, is used to replicate the meshed domain of the targeted region of the exposed cortex. This domain is composed of two media: (1) pial vasculature, and (2) subpial grey matter. The optical properties of the domain in the simulations are selected according to the experimental phases, based on parameters measured during the data acquisition.

Finally, (2) can be written in matrix format (for each pixel $k$, l) and solved using the Moore-Penrose pseudo-inverses of the matrices of the molar extinction coefficients [19], for the three unknowns $\Delta\left[\mathrm{HbO}_{2}\right], \Delta[\mathrm{HHb}]$ and $\Delta[\mathrm{oxCCO}]$.

The last step in the hNIR data processing methodology consists in implementing the spatially-selective, post-processing correction that we developed and validated in Giannoni et al [14] to correct for any underestimation in the quantification of $\mathrm{HbO}_{2}, \mathrm{HHb}$ and oxCCO due to partial volume effects in the MBLL. MC simulations are again used to estimate maps of the distribution of (1) the average partial pathlengths $P L_{k, l, v e s s e l}(\lambda)$ that the detected photons have travelled in the pial vasculature and of (2) the average partial pathlengths $P L_{k, l, g r e y}(\lambda)$ they travelled in the subpial grey matter of the simulated domain (for each wavelength $\lambda$ ). From these and the average total photon pathlengths $P L_{k, l}(\lambda)$ used in (2), two maps of correction factors $C F_{k, l}^{\prime}$ and $C F^{\prime \prime}{ }_{k, l}$ (for each pixel $k, l$ ), are calculated:

$$
\begin{gathered}
C F_{k, l}^{\prime}=\frac{1}{M} \sum_{\mathrm{i}=1}^{M}\left(\frac{P L_{k, l}\left(\lambda_{\mathrm{i}}\right)}{P L_{k, l, \text { vessels }}\left(\lambda_{\mathrm{i}}\right)}\right) \\
C{F^{\prime \prime}{ }_{k, l}=}^{M} \sum_{\mathrm{i}=1}^{M}\left(\frac{P L_{k, l}\left(\lambda_{\mathrm{i}}\right)}{P L_{k, l, \text { grey }}\left(\lambda_{\mathrm{i}}\right)}\right)
\end{gathered}
$$

The two correction maps (averaged between each considered experimental phase and the corresponding baseline) are used to selectively correct the haemodynamic and metabolic maps of $\Delta\left[\mathrm{HbO}_{2}\right], \Delta[\mathrm{HHb}]$ and $\Delta[\mathrm{oxCCO}]$, by weighting for the large differences in the partial pathlengths between pial vasculature and subpial grey matter. This selective correction is performed using the segmented binary map of the FOV utilised during the mesh domain creation as a guide [14]. Thus, for pixels $k, l$ corresponding to the pial vasculature medium in the mask, the following correction is applied to obtain the corrected values $\Delta\left[\mathrm{HbO}_{2}\right]^{*}$ and $\Delta[\mathrm{HHb}]^{*}$ of the changes in concentration of $\mathrm{HbO}_{2}$ and $\mathrm{HHb}$ in the haemodynamic maps only (as no significant presence of CCO is assumed in the blood in the vessels):

$$
\begin{gathered}
\Delta\left[\mathrm{HbO}_{2}\right]_{k, l}^{*}=\Delta\left[\mathrm{HbO}_{2}\right]_{k, l} C F_{k, l}^{\prime} \\
\Delta[\mathrm{HHb}]_{k, l}^{*}=\Delta[\mathrm{HHb}]_{k, l} C F_{k, l}^{\prime}
\end{gathered}
$$

Contrarily, for pixels $k, l$ corresponding to the subpial grey matter medium in the mask, this other correction is applied to both the haemodynamic and the metabolic maps to obtain the corrected values $\Delta\left[\mathrm{HbO}_{2}\right]^{*}, \Delta[\mathrm{HHb}]^{*}$ and $\Delta[\mathrm{oxCCO}]^{*}$ of the changes in concentration of $\mathrm{HbO}_{2}, \mathrm{HHb}$ and oxCCO:

$$
\begin{gathered}
\Delta\left[\mathrm{HbO}_{2}\right]_{k, l}^{*}=\Delta\left[\mathrm{HbO}_{2}\right]_{k, l} C F_{k, l}^{\prime \prime} \\
\Delta[\mathrm{HHb}]_{k, l}^{*}=\Delta[\mathrm{HHb}]_{k, l} C F_{k, l}^{\prime \prime} \\
\Delta[\mathrm{oxCCO}]_{k, l}^{*}=\Delta[\mathrm{oxCCO}]_{k, l} C F^{\prime \prime}{ }_{k, l}
\end{gathered}
$$

\section{VALIDATION ON LIQUID PHANTOMS}

The hNIR system was tested and validated on homogeneous liquid optical phantoms based on blood, Intralipid and yeast, in order to assess its ability and performances to simultaneously measure and resolve changes in the concentrations of the two forms of haemoglobin and the oxidative state of CCO. Liquid optical phantoms based on blood and Intralipid are commonly used in NIRS [20], [21] and bNIRS [22] to validate the capacity of an instrument to retrieve correct information about haemoglobin oxygenation, due to the fitness of such phantoms for mimicking the optical properties of biological tissues. The oxygenation and deoxygenation of the blood in the liquid phantoms can be performed in various ways, such as by bubbling oxygen gas $\left(\mathrm{O}_{2}\right)$ for oxygenating the blood and by bubbling nitrogen gas $\left(\mathrm{N}_{2}\right)$ for deoxygenating it. Furthermore, the addition of yeast to this type of phantoms as a deoxygenating agent has been recently demonstrated to be an effective way to validate the ability of a bNIRS system to also concurrently resolve changes in the concentration of oxCCO [22]. This is because yeast consumes oxygen using the same aerobic process of human tissues, including the ETC with the oxidation-reduction of $\mathrm{CCO}$. Thus, the presence and variations in the concentration of oxCCO in the yeast of the liquid phantoms can be detected during blood oxygenation and deoxygenation.

\section{A. Liquid phantoms preparation}

The recipes and preparation of the liquid optical phantoms used for the experiments described here are based on procedures by F. Lange et al [22]. Two phantoms, of $1.5 \mathrm{~L}$ each, were prepared: one (Phantom 1) containing deionised water, Intralipid, phosphate-buffered saline (PBS) and blood, while the other (Phantom 2) including the same components with the addition of yeast. Both liquid phantoms were contained in a metallic box of $27 \times 15 \times 16 \mathrm{~cm}$. The inner surface of the box was covered in black absorbing paint to minimise reflection: this was done to mitigate boundaries effect and thus approximate the liquid phantoms to semi-infinite media.

First, a solution of $1.4 \mathrm{~L}$ of deionised water, $50 \mathrm{mM}$ PBS 
(Sigma-Aldrich PBS P3813) and $75 \mathrm{~g}$ of Intralipid 20\% (Fresenius Kabi Intralipid 20\%) was poured into the box, which was then constantly stirred and kept homogeneous using a magnetic stirrer and also maintained at a temperature of about $36.5^{\circ}-37^{\circ}$ using a hot plate. The use of deionised water and Intralipid had the purpose of mimicking the absorption and scattering properties of two of the main components of biological tissues, i.e. water and lipid [23], [24], while PBS was added to achieve and maintain a physiological pH (approximately 7.2-7.4) in the liquid phantoms. Afterwards, $25 \mathrm{~mL}$ of blood were added to both phantoms using a syringe, to replicate the absorption properties of haemoglobin. For Phantom 2, $10 \mathrm{~g}$ of yeast were then added to achieve deoxygenation during the experimental procedure.

Oxygenation via $\mathrm{O}_{2}$ bubbling (for both phantoms) and deoxygenation via $\mathrm{N}_{2}$ bubbling (only for Phantom 1) were induced using industrial gas tanks connected to air bubbling stones, that were positioned at the bottom of the metallic box.

The most relevant physiological parameters of both liquid phantoms were constantly monitored during the experiments, in particular: (1) the temperature of the mixtures, using a temperature probe; (2) the $\mathrm{pH}$ of the mixtures, using a $\mathrm{pH}$ sensor; and (3) the content of dissolved oxygen (DO) in the mixtures, using a chemical oxygen sensor.

\section{B. Experimental procedure and data collection}

The same data acquisition process is conducted with hNIR, consisting of imaging separately both phantoms during alternating phases of deoxygenation ( 3 phases) and re-oxygenation (2 phases). In Phantom 1, deoxygenation is obtained via bubbling of $\mathrm{N}_{2}$, while in Phantom 2 via the yeast. This approach allows separation of the signals of haemoglobin, present in both phantoms, from the signal of CCO, present only in Phantom 2, thus assessing whether the changes in the concentration of oxCCO measured by hNIR are effectively generated by the optical signature of this chromophore and do not arise from crosstalk.

Only the 8 NIR spectral bands of hNIR were used (784, 800, $818,835,851,868,881$ and $894 \mathrm{~nm}$ ), since both phantoms are homogeneous and no spatial features were to be resolved, therefore the addition of visible light was not necessary.

The illumination fibre and FOV of hNIR were positioned over a region of the liquid phantoms that was distant enough from both the stirrer and the gas diffusers, in order to minimise motion artefacts in the collected hyperspectral data.

Each experimental procedure started after the mixtures of the phantoms reached homogeneity and stable temperature $\left(36.5^{\circ}\right.$ $\left.37^{\circ}\right)$. Firstly, each phantom was oxygenated by bubbling $\mathrm{O}_{2}(3$ $\mathrm{L} / \mathrm{min}$ ) to a DO equal to about $100 \%$ : this phase corresponded to the baseline condition for the data collection. After baseline, a phase of deoxygenation followed (DO less than 10\%), induced either by bubbling $\mathrm{N}_{2}$ (for Phantom 1 , at $7 \mathrm{~L} / \mathrm{min}$ ) or by waiting for yeast to deoxygenate the mixture (for Phantom 2). Data collection for the deoxygenation phase started after DO had stabilised in time. Following HSI during the deoxygenation phase, both phantoms were re-oxygenated by bubbling $\mathrm{O}_{2}$ again, to reach a DO that was approximately equal to the baseline condition (DO standing at about 100\%). As for the previous phase, data acquisition was again started after DO had reached stability in time. Two additional deoxygenation phases and one re-oxygenation phase in between the two were repeated before concluding both procedures. For Phantom 2, the two extra deoxygenation phases were obtained by switching off the $\mathrm{O}_{2}$ bubbling and waiting for the yeast in the mixture to consume again the oxygen.

During the initial baseline and each of the five following deoxygenation and re-oxygenation phases, 20 hypercubes were acquired every time for $1 \mathrm{~min}$ (for both phantoms). Data collection was not performed during the dynamic changes in the oxygenation levels of the various phases, due to formation of bubbles on the surface of the phantoms, distorting the FOV and risking staining of the objective mirrors. The bubbles disappeared by the time a stationary DO level was reached.

Time-averaging was performed on all the hypercubes in each phase (both baseline and the five deoxygenation and re-oxygenation phases). Thus, one final time-averaged hypercube over the entire recording time $(1 \mathrm{~min})$ was obtained for each phase.

\section{In Vivo APPLICATION ON MiCE}

The hNIR system was used to perform in vivo HSI of the exposed cerebral cortex of three anesthetised healthy mice breathing a series of normoxic, hyperoxic and hypoxic gas mixtures of oxygen and nitrogen, until termination of the animals (still under anaesthesia) during anoxia, at the end of the procedure. These oxygen manipulations were chosen as they produce significant changes in haemoglobin oxygenation and in the redox states of $\mathrm{CCO}$, as extensively demonstrated in the literature with the application of bNIRS [9]. The different degrees of oxygenation to the brain tissue of the mice were established by manipulating their fractions of inspired oxygen $\left(\mathrm{FiO}_{2}\right)$.

\section{A. Animal preparation}

The three imaged mice (inbred strain $\mathrm{C} 57 \mathrm{bL} / 6$ of average weight of $\sim 22.3 \mathrm{~g}$ ) were anesthetised with 1.5-2\% isoflurane in room air and then placed on a homoeothermic electric-heating mat that maintained the mice at constant temperature (36.5-37 ${ }^{\circ} \mathrm{C}$ ). The scalp of each mouse was surgically removed and a partial craniotomy was performed over the right hemisphere without damaging the dura, creating an exposed cortical window of about 4-5 $\mathrm{mm}$ in diameter. The dura was moistened and cleaned with saline solution, before applying a circular glass coverslip over a ring of Vaseline, to prevent evaporation during imaging and contamination of the exposed cortex from ambient air. Finally, the head of each mouse was stabilised using a titanium bar and dental cement to prevent movement artefacts in the collected images. Anaesthesia was maintained for the entire experimental procedure until termination of the animals.

All procedures were performed in accordance with the UK Home Office Animals (Scientific Procedures) Act (1986) and the EU Directive 2010/63/EU.

\section{B. Experimental procedure and data collection}

The experimental imaging procedure performed on each 
mouse consisted of the following series of consecutive oxygendependent phases: 1) normoxic baseline $\left(\mathrm{FiO}_{2}=21 \%\right)$; 2) hyperoxia $\left(\mathrm{FiO}_{2}=93 \%\right)$; 3) normoxia $\left(\mathrm{FiO}_{2}=21 \%\right)$; 4) hypoxia $\left.\left(\mathrm{FiO}_{2}=10 \%\right) ; 5\right)$ normoxia $\left.\left(\mathrm{FiO}_{2}=21 \%\right) ; 6\right)$ anoxia followed by death of the animal $\left(\mathrm{FiO}_{2}=0 \%\right)$. For each experimental phase, hNIR was run for all its 11 spectral bands $(600,630,665,784$, $800,818,835,851,868,881$ and $894 \mathrm{~nm}$ ).

During the initial normoxic baseline, 40 hypercubes were collected for a total acquisition time of $2 \mathrm{~min}$. Subsequently, for each of the following phases, 100 hypercubes were then acquired for $5 \mathrm{~min}$. Time-averaging was performed on all the 40 hypercubes of the normoxic baseline over the entire integration time of 2 min to obtain a single time-averaged baseline hypercube. For the 100 hypercubes of each oxygen-dependent phase, time-averaging was performed for every 10 hypercubes in the 5-min acquisition time interval, obtaining 10 averaged hypercubes per each imaged condition: each averaged hypercube corresponds to a time window of $30 \mathrm{~s}$ of hyperspectral recording on the exposed cerebral cortex.

\section{RESUlts}

\section{A. Results of the phantoms validation}

All the haemodynamic and metabolic maps reconstructed with hNIR from (2) for each phase and for both liquid phantoms provide no spatial features to be resolved, as expected since they are composed of homogeneous liquid mixtures.

Spatial averages $<\Delta\left[\mathrm{HbO}_{2}\right]>,<\Delta[\mathrm{HHb}]>$ and $<\Delta[\mathrm{oxCCO}]>$ of the relative changes in the concentrations of $\mathrm{HbO}_{2}, \mathrm{HHb}$ and oxCCO are then calculated from the reconstructed maps, to analyse and assess the measured responses from each phantom in all the experimental phases. This is done by spatially averaging the relative changes in concentrations $\Delta\left[\mathrm{HbO}_{2}\right], \Delta[\mathrm{HHb}]$ and $\Delta[\mathrm{oxCCO}]$ in every map across all the pixels of the whole FOV (due to the homogeneity of the phantoms), for each phase. Thus, average quantified values of the haemodynamic and metabolic responses of both liquid phantoms in each experimental phase are obtained. These values are reported in Fig. 4 against the corresponding DO level of each experimental phase (Fig. 4a for Phantom 1 and Fig. 4b for Phantom 2).

The spectroscopic analysis on the overall average changes in $\Delta\left[\mathrm{HbO}_{2}\right], \Delta[\mathrm{HHb}]$ and $\Delta[\mathrm{oxCCO}]$ first shows that hNIR is able to detect and reconstruct the expected temporal trends of the changes in the concentrations of $\mathrm{HbO}_{2}$ and $\mathrm{HHb}$ during all the series of deoxygenation and re-oxygenation events, for both Phantom 1 and Phantom 2. During the deoxygenation events, where the DO level significantly drops compared to baseline, decreases in $\left\langle\Delta\left[\mathrm{HbO}_{2}\right]>(-36.02 \pm 1.84 \mu \mathrm{M}\right.$ for Phantom 1 and $33.23 \pm 1.01 \mu \mathrm{M}$ for Phantom 2 , on average across all deoxygenation phases) and increases in $\langle\Delta[\mathrm{HHb}]\rangle(35.67 \pm 1.58 \mu \mathrm{M}$ for Phantom 1 and $34.81 \pm 0.73 \mu \mathrm{M}$ for Phantom 2, on average across all deoxygenation phases) are estimated for both phantoms, while no significant changes occur in either $\left\langle\Delta\left[\mathrm{HbO}_{2}\right]>\right.$ $(1.31 \pm 0.08 \mu \mathrm{M}$ for Phantom 1 and $0.92 \pm 0.19 \mu \mathrm{M}$ for Phantom 2 , on average across all re-oxygenation phases) or $\langle\Delta[\mathrm{HHb}]>$ $(-2.96 \pm 0.51 \mu \mathrm{M}$ for Phantom 1 and $-2.65 \pm 0.08 \mu \mathrm{M}$ for Phantom 2 , on average across all re-oxygenation phases) during the re-
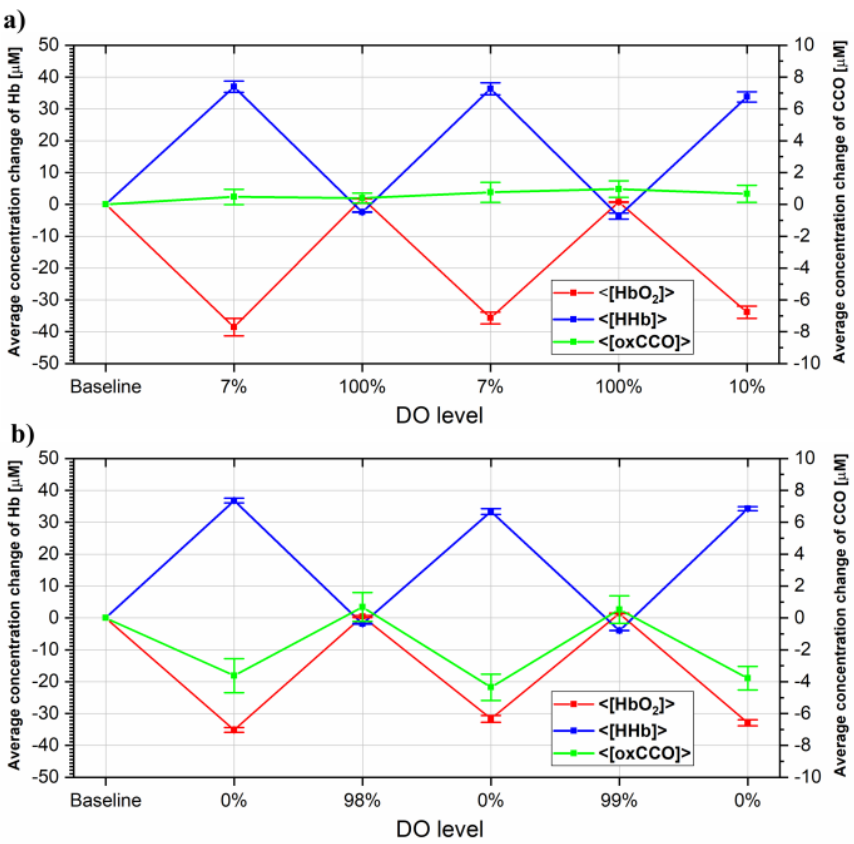

Fig. 4. Spectroscopic analysis over the whole FOVs of the maps of the phantoms imaged with hNIR, showing the average changes $\left\langle\Delta\left[\mathrm{HbO}_{2}\right]\right\rangle$ (red), $<\Delta[\mathrm{HHb}]>$ (blue) and $<\Delta$ [oxCCO] $>$ (green) during all the phases of the validation procedures. The results for Phantom 1, including only blood, are shown in (a), while (b) shows the results for Phantom 2, made of blood and yeast.

oxygenation events, when DO levels rise back to the baseline equivalents. Secondly, Fig. 4a shows that, for Phantom 1, no significant metabolic response due to changes in $\langle\Delta[\mathrm{oxCCO}]\rangle$ is reconstructed in any of the phases, as the spatial averages of the relative changes in concentration of oxCCO are all negligible $(0.93 \pm 0.49 \mu \mathrm{M}$ on average across all phases $)$. Contrariwise, metabolic responses of $\mathrm{CCO}$ to the deoxygenation events are measured for Phantom 2, as expected since the presence of yeast in the mixture. As for the haemodynamic responses of $\mathrm{HbO}_{2}$ and $\mathrm{HHb}$, the spatial averages $<\Delta[\mathrm{oxCCO}]>$ in all the phases in Phantom 2 also follow the projected trends, with the concentration of $\mathrm{CCO}$ decreasing during deoxygenation due to oxygen depletion $(-4.02 \pm 0.79 \mu \mathrm{M}$ on average across all deoxygenation phases), and showing negligible changes during re-oxygenation $(0.85 \pm 0.89 \mu \mathrm{M}$ on average across all re-oxygenation phases), due to return to baseline levels of DO.

\section{B. Results of the in vivo application}

An example for one mouse of the corrected haemodynamic and metabolic maps of the relative changes $\Delta\left[\mathrm{HbO}_{2}\right], \Delta[\mathrm{HHb}]$ and $\Delta[\mathrm{oxCCO}]$ in the concentrations of $\mathrm{HbO}_{2}, \mathrm{HHb}$ and oxCCO after applying the spatially-selective, post-processing correction is reported in Fig. 5. The maps in Fig. 5 are not depicted for all the 10 time windows of each phase (50 maps in total), but only for 4 of them, evenly spaced every $60 \mathrm{~s}$ in the 5-min acquisition time of each experimental phase. This was done in order to summarise and show the dynamic changes in the concentrations of the three targeted chromophores throughout the whole experiment. The full set of maps in all the time windows of all the phases are reported in their entirety as videos in the attached supplementary multimedia material (including both 
a)

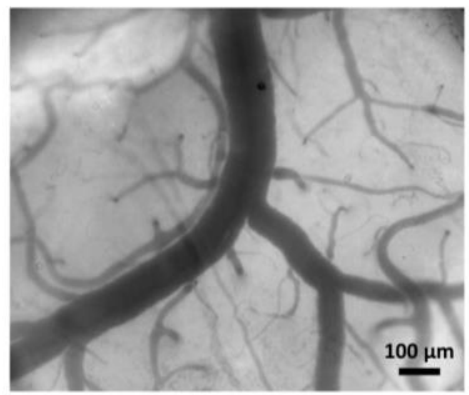

b)

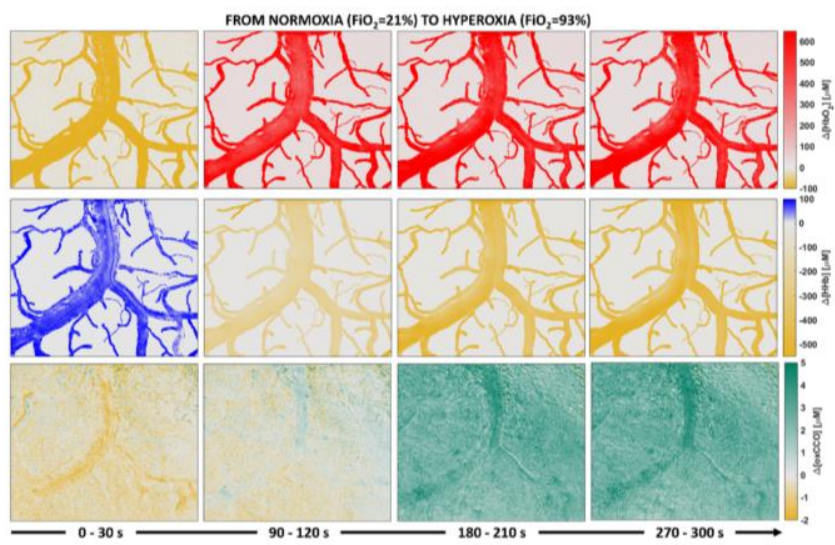

FROM NORMOXIA (FiO $=21 \%)$ TO HYPOXIA (FiO $=10 \%)$

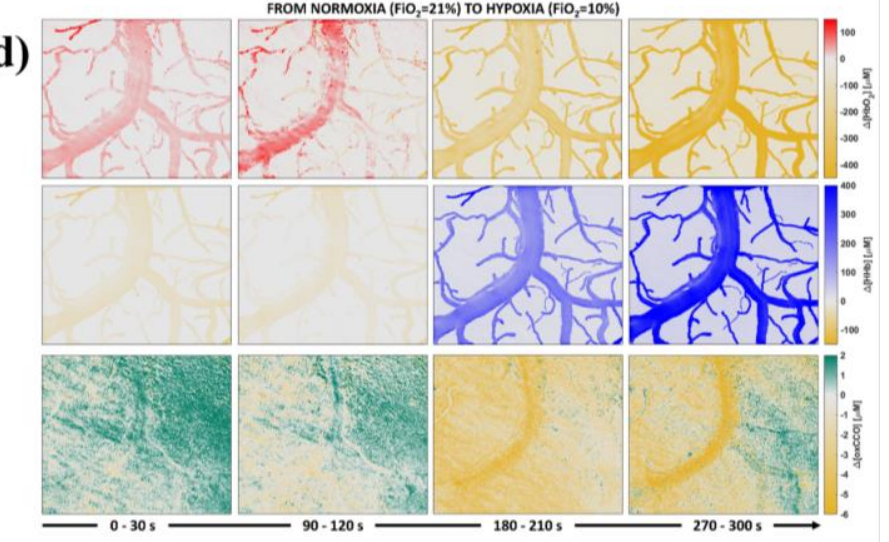

FROM NORMOXIA (FiO $=21 \%)$ TO ANOXIA $\left(\mathrm{FFO}_{2}=0 \%\right.$ c)

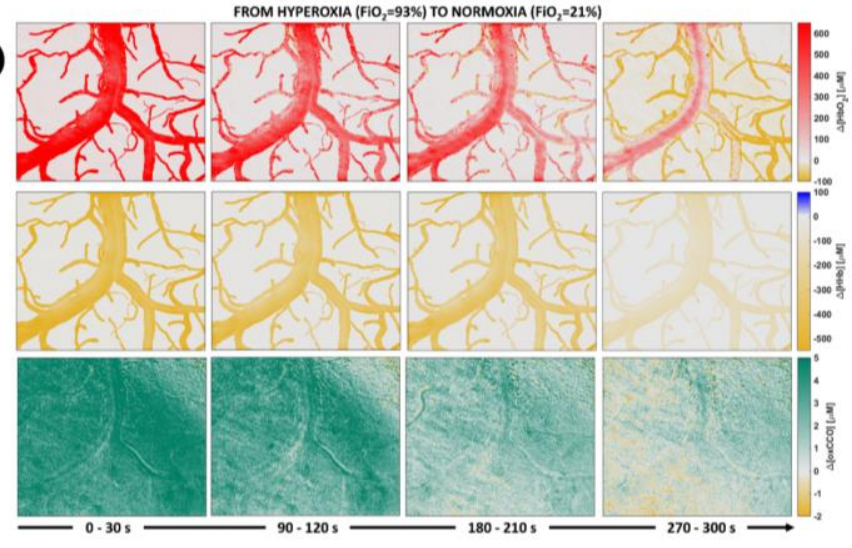

e)

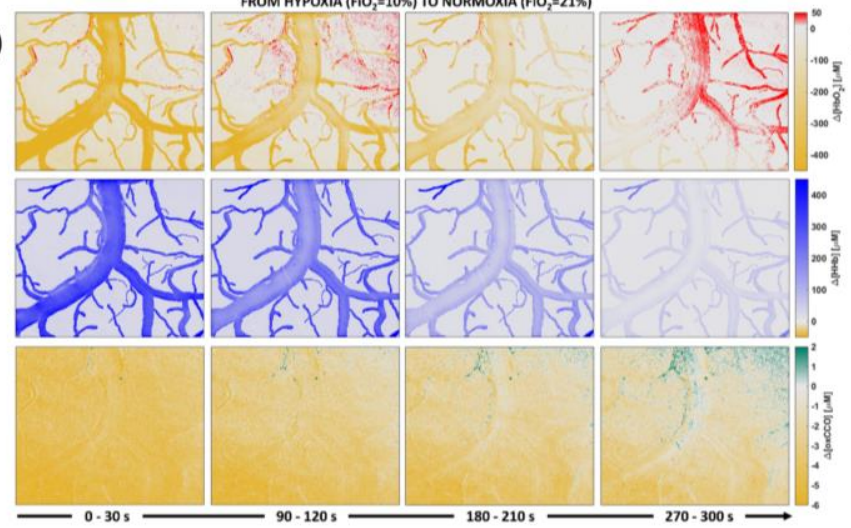

f)

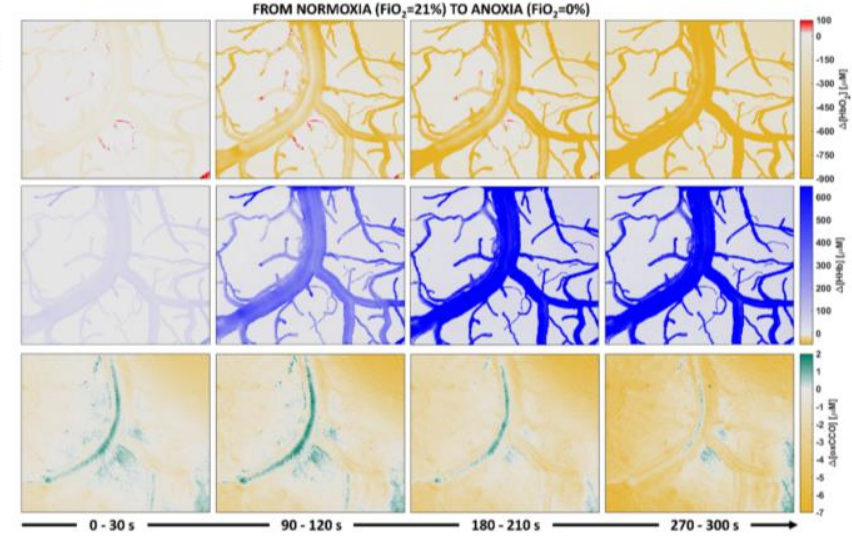

Fig. 5. Corrected maps of the relative changes in concentration of $\mathrm{HbO}_{2}$ (top row), $\mathrm{HHb}$ (middle row) and oxCCO (bottom row) on the exposed cortex of a mouse, obtained with hNIR after spatially-selective, post-processing correction. Each map corresponds to an averaged time window of $30 \mathrm{~s}$ during a 5 -min acquisition interval. The maps are compared with a corresponding greyscale image of the FOV (a) obtained with the imaging side of hNIR and broadband illumination. All the phases of the experiments are reported: (b) from normoxia to hyperoxia; (c) from hyperoxia to normoxia; (d) from normoxia to hypoxia; (e) from hypoxia to normoxia; (f) from normoxia to anoxia.

corrected and uncorrected results), showing full temporal resolution capacity of the system. These will be available at http://ieeexplore.ieee.org.

The haemodynamic maps of $\mathrm{HbO}_{2}$ and $\mathrm{HHb}$ on the exposed cortex reconstructed with hNIR present high spatial resolution and image contrast, across all the experimental phases, allowing the visualisation of both major and minor vasculature (about $100-120 \mu \mathrm{m}$ and $10-20 \mu \mathrm{m}$ in diameter, respectively), compared to the greyscale image of the FOV (Fig. 5a). The haemoglobin changes are largely constrained and confined within the pial vasculature. The large and predominant haemodynamic responses of $\mathrm{HbO}_{2}$ and $\mathrm{HHb}$ in the pial vasculature are correctly localised and reproduce the expected temporal trends for the corresponding experimental phases: (1) an increase in $\Delta\left[\mathrm{HbO}_{2}\right]$ and a decrease in $\Delta[\mathrm{HHb}]$ during hyperoxia; (2) reduction in $\Delta\left[\mathrm{HbO}_{2}\right]$ and an increment in $\Delta[\mathrm{HHb}]$ during hypoxia and anoxia; (3) a return to near-baseline values $\left(\Delta\left[\mathrm{HbO}_{2}\right]\right.$ and $\Delta[\mathrm{HHb}]$ close to zero) during normoxia. Smaller haemodynamic responses are also reconstructed in the surrounding subpial grey matter, consistent with the changes in oxygen saturation and perfusion of the extravascular cerebral tissue (following the same trends of the pial vasculature). These responses appear generally homogeneous across the whole subpial tissue.

The time dynamics of the changes in concentrations of $\mathrm{HbO}_{2}$ and $\mathrm{HHb}$ during the various phases is also successfully captured with the hNIR system, providing suitable temporal resolution 
for all the physiological processes involved (this emerges more explicitly from the video in the attached supplementary multimedia material). The two phases of return to normoxia after hyperoxia and hypoxia still present remnants of the haemodynamic responses, with non-null values of $\Delta\left[\mathrm{HbO}_{2}\right]$ and $\Delta[\mathrm{HHb}]$ and some contrast between vasculature and surrounding tissue in the maps: this phenomenon could have physiological causes, as brain oxygenation may require more than $5 \mathrm{~min}$ to completely return to baseline levels, especially after hypoxia.

The metabolic maps of oxCCO present lower spatial resolution and image contrast than the haemodynamic maps, with the metabolic response being spread across the whole FOV: the major vasculature is resolved during all the experimental phases, with the minor vessels becoming visible only during the last time windows of anoxic death. Nonetheless, localisation of the metabolic response correlated to the changes in the concentration of oxCCO is correctly achieved in the extravascular cerebral tissue throughout the whole experiment, although spurious measured changes $\Delta[\mathrm{oxCCO}]$ are reconstructed also in the main pial vasculature (where CCO presence should be negligible). Generally, the metabolic maps show the expected temporal trends of the metabolic response of oxCCO during all the conditions: (1) increase in $\Delta[\mathrm{oxCCO}]$ during hyperoxia; (2) decrease in $\Delta[\mathrm{oxCCO}]$ during hypoxia and anoxia; (3) return to quasi-null values of $\Delta[\mathrm{oxCCO}]$ during the normoxic events.

Spectroscopic analysis on all the corrected reconstructed maps of $\Delta\left[\mathrm{HbO}_{2}\right], \Delta[\mathrm{HHb}]$ and $\Delta[\mathrm{oxCCO}]$ on the exposed cortex of the mice is also conducted by targeting two specific regions of interest (ROI) on the imaged FOV, each composed of $200 \times 200$ pixels and corresponding to cortical areas of about $85 \times 85 \mu \mathrm{m}$ : (1) the first ROI is entirely located on a region encompassing only the major vasculature; (2) the second ROI is centred on a section of subpial grey matter, where major vessels are not present. The spectroscopic analysis consists of spatially averaging the relative changes $\Delta\left[\mathrm{HbO}_{2}\right]_{k, l}, \Delta[\mathrm{HHb}]_{k, l}$ and $\Delta[\mathrm{ox}-$ $\mathrm{CCO}]_{k, l}$ across all the pixels $k, l$ of the selected ROIs in the maps. The temporal average variations $\left\langle\Delta\left[\mathrm{HbO}_{2}\right]>,\langle\Delta[\mathrm{HHb}]>\right.$ and $\langle\Delta$ [oxCCO] $>$ in the two ROIs are obtained this way, for each time window of the experiments. An example of the results of the spectroscopic analysis of $\Delta\left[\mathrm{HbO}_{2}\right], \Delta[\mathrm{HHb}]$ and $\Delta[\mathrm{ox}-$ $\mathrm{CCO}$ ] are shown in Fig. 6 (for the same mouse of Fig. 5). Each data point in the graphs, corresponding to a single time window, is a mean across the ROI with standard deviation.

The temporal trends of the average changes $\left\langle\Delta\left[\mathrm{HbO}_{2}\right]>\right.$, $<\Delta[\mathrm{HHb}]>$ and $<\Delta[\mathrm{oxCCO}]>$ in both ROIs confirm the results of the monitoring of haemodynamics and metabolism on the exposed cortex shown by the reconstructed maps, as well as the ability of hNIR to correctly track and quantify changes in the concentrations of $\mathrm{HbO}_{2}, \mathrm{HHb}$ and oxCCO. The values of $<\Delta\left[\mathrm{HbO}_{2}\right]>$ and $<\Delta[\mathrm{HHb}]>$ over time follow the expected haemodynamic responses of haemoglobin to hyperoxia, hypoxia and anoxia in both ROIs. For the ROI located in the pial vasculature: (1) during hyperoxia, $<\Delta\left[\mathrm{HbO}_{2}\right]>$ peaks at a maximum average value of $483.07 \pm 11.16 \mu \mathrm{M}$ (mean across all mice), while a maximum decrease of $-334.46 \pm 8.92 \mu \mathrm{M}$ in $\langle\Delta[\mathrm{HHb}]>$ (mean across all mice) is identified. Both responses occur within 270-330 s since the start of the experiment. (2) During
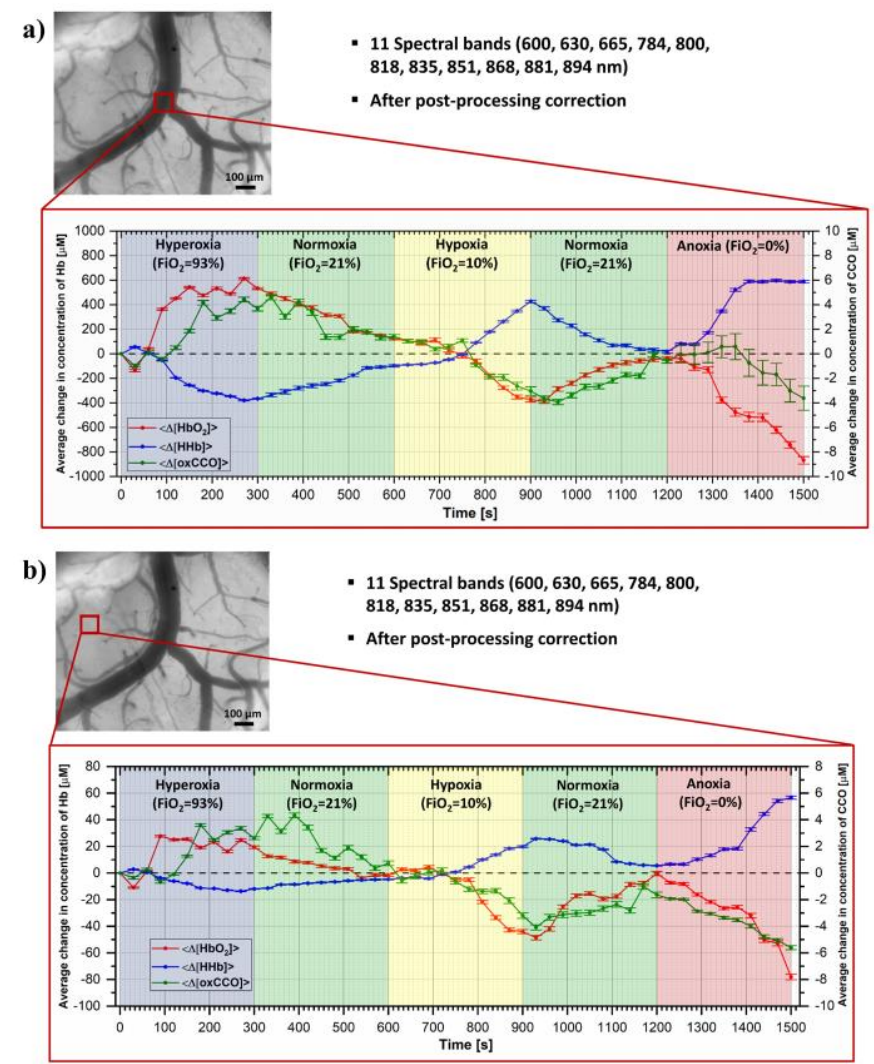

Fig. 6. Spectroscopic analysis in two ROIs of the corrected maps of the exposed cortex of one mouse obtained by hNIR, showing the temporal average changes $\left\langle\Delta\left[\mathrm{HbO}_{2}\right]>\right.$ (red), $<\Delta[\mathrm{HHb}]>$ (blue) and $\langle\Delta[$ oxCCO $]>$ (green), during all the phases of the experiments. The first ROI (a) lies all within a major vessel, while the second (b) includes only the surrounding brain tissue.

hypoxia, $<\Delta\left[\mathrm{HbO}_{2}\right]>$ decreases to a minimum of $-388.11 \pm 18.08$ $\mu \mathrm{M}$ in the interval 720-750 s and $<\Delta[\mathrm{HHb}]>$ increases to a maximum of $365.01 \pm 16.92 \mu \mathrm{M}$ in the interval 690-750 s (means across all mice), whereas during anoxia $<\Delta\left[\mathrm{HbO}_{2}\right]>$ drops to a minimum of $-823.11 \pm 46.92 \mu \mathrm{M}$ in the interval $1470-1500 \mathrm{~s}$ and $<\Delta[\mathrm{HHb}]>$ peaks to a maximum of $538.68 \pm 26.92 \mu \mathrm{M}$ in the interval 1440-1500 s (means across all mice). (3) During the normoxic events, returns to quasi-baseline values are reported for both $\mathrm{HbO}_{2}$ and $\mathrm{HHb}$, consistently with the premises of the experiments $\left(<\Delta\left[\mathrm{HbO}_{2}\right]>\right.$ stabilising around $15.80 \pm 5.35 \mu \mathrm{M}$ and $-5.25 \pm 4.85 \mu \mathrm{M}$, while $<\Delta[\mathrm{HHb}]>$ returning to $-7.57 \pm 6.21$ $\mu \mathrm{M}$ and to $7.52 \pm 7.05 \mu \mathrm{M}$, for the first and second normoxic events, respectively across all mice).

The changes in $\mathrm{HbO}_{2}$ and $\mathrm{HHb}$ appear larger by $100-200$ times in the ROI located in the vasculature than in the ROI centred over extravascular grey matter, as expected due to the higher presence of haemoglobin and greater changes in oxygenation in the vessels. For the ROI located in the subpial grey matter: (1) during hyperoxia, $<\Delta\left[\mathrm{HbO}_{2}\right]>$ increases to a maximum of $28.32 \pm 2.43 \mu \mathrm{M}$ (mean across all mice), while $<\Delta[\mathrm{HHb}]>$ has a maximum drop of $-18.68 \pm 1.49 \mu \mathrm{M}$ (mean across all mice). (2) During hypoxia, $<\Delta\left[\mathrm{HbO}_{2}\right]>$ decreases to a minimum of $51.05 \pm 6.18 \mu \mathrm{M}$ and $\langle\Delta[\mathrm{HHb}]>$ increases to a maximum of $35.57 \pm 4.35 \mu \mathrm{M}$ (means across all mice), whereas during anoxia $<\Delta\left[\mathrm{HbO}_{2}\right]>$ drops to a minimum of $-82.97 \pm 10.08 \mu \mathrm{M}$ and $<\Delta[\mathrm{HHb}]>$ peaks to a maximum of $61.63 \pm 6.43 \mu \mathrm{M}$ (means 
across all mice). (3) During the normoxic events, both subpial $\mathrm{HbO}_{2}$ and $\mathrm{HHb}$ return again to quasi-baseline levels.

In general, the haemodynamic responses of both the two forms of haemoglobin show greater magnitude during the phases of reduced oxygenation, i.e. hypoxia and anoxia, compared to less significant changes in $\left\langle\Delta\left[\mathrm{HbO}_{2}\right]>\right.$ and $\langle\Delta[\mathrm{HHb}]\rangle$ during hyperoxia (for both ROIs). The haemodynamic responses of $\mathrm{HbO}_{2}$ in the pial vasculature present an initial dip (on average equal to $-103.83 \pm 7.12 \mu \mathrm{M}$, across all mice) in the first time window of hyperoxia (0-30 s). Furthermore, the haemodynamic response of $\mathrm{HHb}$ in the pial vasculature during anoxia reaches an approximately stable plateau after the death of the mouse (around 1350-1410 s).

The temporal trends of $\langle\Delta[\mathrm{oxCCO}]>$ in the ROI including only extravascular brain tissue is also consistent with the expected metabolic responses of the exposed cortex to hyperoxia, hypoxia and anoxic death, as well as to the two returns to normoxia (close to zero changes compared to baseline). During hyperoxia, $<\Delta[$ oxCCO $]>$ rises to a maximum of $4.41 \pm 0.98 \mu \mathrm{M}$ within 330-390 s (mean across all mice). During hypoxia, it drops to a minimum of $-4.15 \pm 1.19$ within $900-990 \mathrm{~s}$ (means

\section{(a) PIAL VASCULATURE}
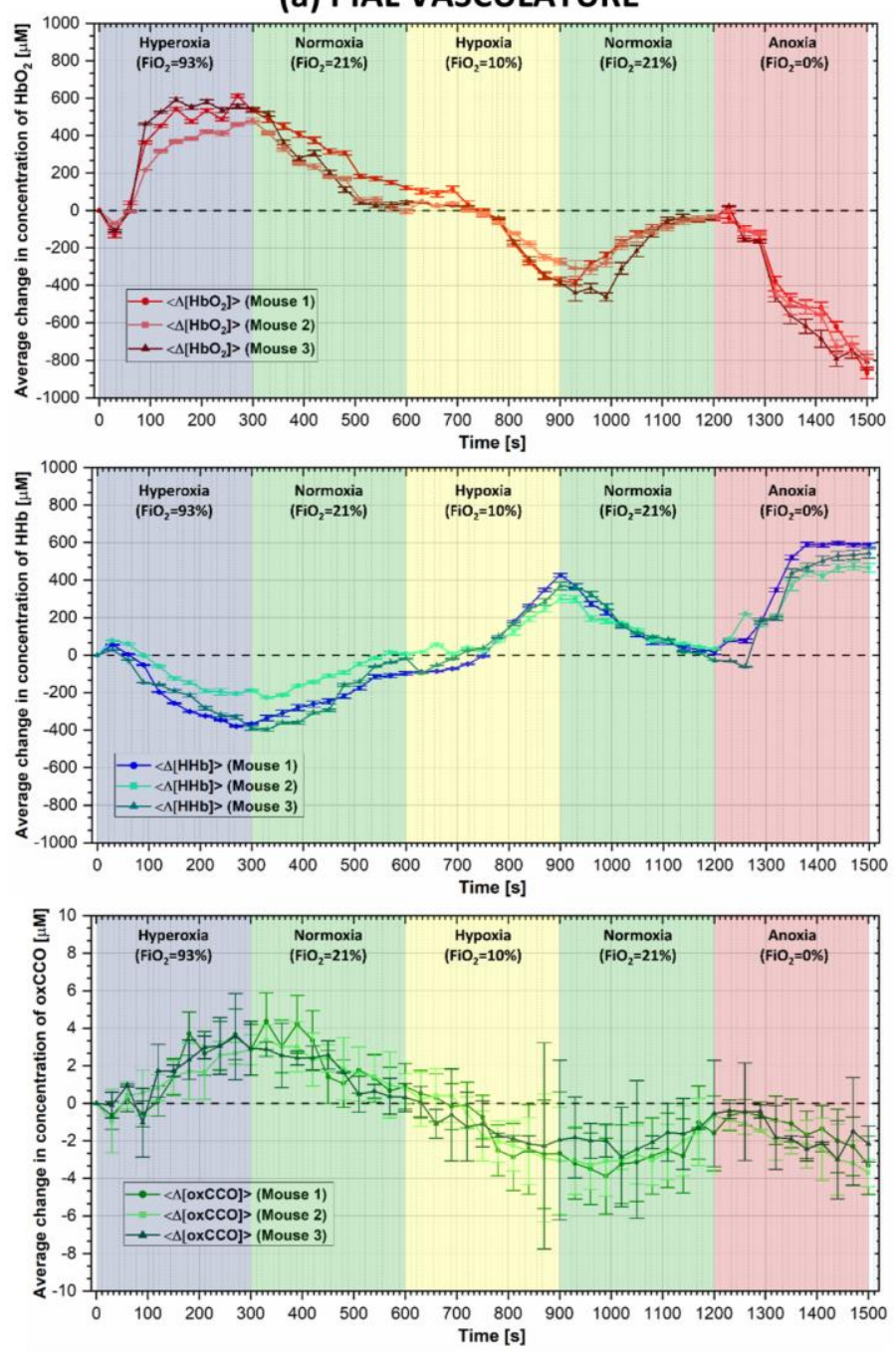

across all mice), whereas during anoxia $<\Delta[$ oxCCO $]>$ is reduced to a minimum of $-6.06 \pm 1.85$ within $1470-1500 \mathrm{~s}$ (means across all mice). (3) During the normoxic events, $<\Delta[\mathrm{oxCCO}]>$ returns to quasi-baseline values (stabilising around $-0.04 \pm 0.61$ $\mu \mathrm{M}$ and $-0.86 \pm 0.97 \mu \mathrm{M}$, for the first and second normoxic events, respectively across all mice). The metabolic response of oxCCO generally presents a delay of 30-60 s in its inception and peaking compared to the haemodynamic responses of haemoglobin, in particular during hyperoxia and hypoxia. The presence of spurious measured changes in the concentration of oxCCO in the ROI comprising only the pial vasculature is also apparent: these values of $<\Delta[\mathrm{oxCCO}]>$ are of the same order of magnitude of the corresponding spatial averages in the ROI outside of the vessels, until they start to differ more significantly after the death of the mice. The spurious measured changes in oxCCO in the pial vasculature also follow similar time dynamics to the metabolic response in the subpial grey matter in the whole experiment. The spurious changes $<\Delta$ [oxCCO] $>$ in the pial vasculature during anoxic death are characterised by a larger variance, as demonstrated by the values of the corresponding standard deviations (around \pm 2.01 across all mice).

\section{(b) SUBPIAL GREY MATTER}
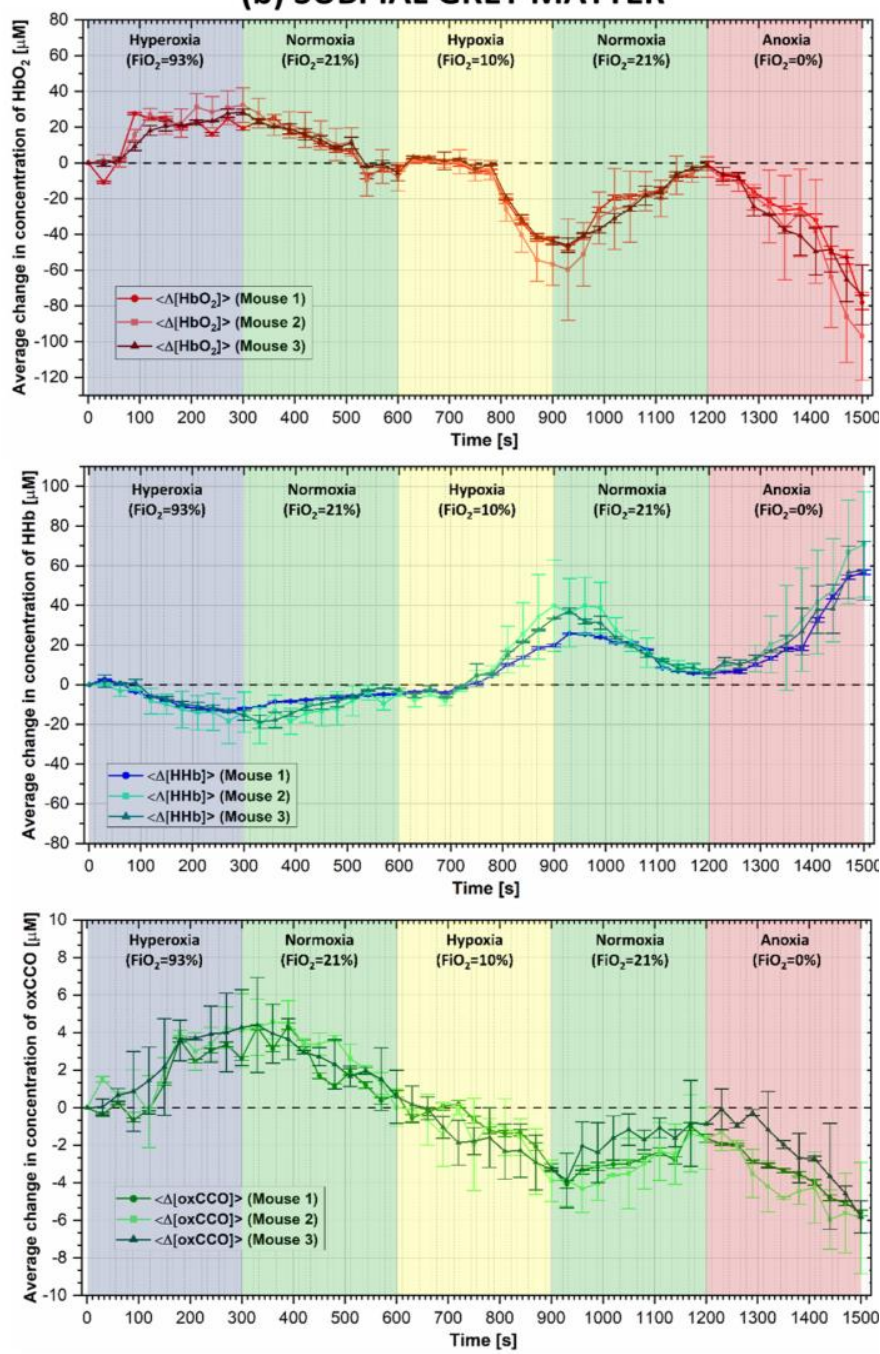

Fig. 7. Medium-wise spectroscopic analysis on the corrected maps of the exposed cortexes of the three mice, showing the temporal average changes $<\Delta\left[\mathrm{HbO}_{2}\right]>$ (top row), $<\Delta[\mathrm{HHb}]>$ (middle row) and $<\Delta[\mathrm{oxCCO}]>$ (bottom row) over the entire pial vascular medium (a) and over the whole subpial grey matter medium (b), during all the phases of the experiment. 
Finally, a group, medium-wise spectroscopic analysis is conducted across all the three imaged mice to compare the variability of the results between the animals. Using the binary masks generated during the creation of the meshed domain for the MC simulations [14], that differentiate the FOVs of the exposed cortexes between vasculature and surrounding subpial tissue, temporal average variations of $\left\langle\Delta\left[\mathrm{HbO}_{2}\right]\right\rangle,\langle\Delta[\mathrm{HHb}]\rangle$ and $<\Delta[$ oxCCO $]>$ were calculated by spatially averaging the changes $\Delta\left[\mathrm{HbO}_{2}\right]_{k, l}, \Delta[\mathrm{HHb}]_{k, l}$ and $\Delta[\text { oxCCO }]_{k, l}$ in the concentrations of $\mathrm{HbO}_{2}, \mathrm{HHb}$ and oxCCO across all the pixels $k, l$ belonging to each medium, during each time window of the experiments. These sets of temporal trends, each having one curve for the vasculature and one for the surrounding subpial tissue, were then plotted for all the three mice, to compare the overall variations of $\left\langle\Delta\left[\mathrm{HbO}_{2}\right]>,<\Delta[\mathrm{HHb}]>\right.$ and $\langle\Delta[\mathrm{oxCCO}]>$ in the vessels and in the subpial grey matter, for the whole study.

Fig. 7 shows the results of the medium-wise spectroscopic analysis for all the three subject mice and for the hyperspectral datasets reconstructed and corrected with hNIR. The temporal trends of $\left\langle\Delta\left[\mathrm{HbO}_{2}\right]\right\rangle,\langle\Delta[\mathrm{HHb}]>$ and $\langle\Delta[$ oxCCO $]>$ appear similar between the three subject mice, with no significantly large variability in the reconstructed haemodynamic and metabolic responses of $\mathrm{HbO}_{2}, \mathrm{HHb}$ and oxCCO across the subjects. The mean differences between the time trends of the three mice ranges at about $3.36-25.53 \%$ for $\left\langle\Delta\left[\mathrm{HbO}_{2}\right]\right\rangle, 5.36-33.87 \%$ for $<\Delta[\mathrm{HHb}]>$, and $1.52-17.86 \%$ for $\langle\Delta[\mathrm{oxCCO}]>$, for the results for the entire pial vasculature (Fig 7a), whereas at about 1.12$11.42 \%$ for $\left\langle\Delta\left[\mathrm{HbO}_{2}\right]>, 2.12-16.54 \%\right.$ for $\langle\Delta[\mathrm{HHb}]>$, and $4.53-$ $21.25 \%$ for $\langle\Delta[\mathrm{oxCCO}]>$, for the results including only extravascular tissue (Fig. 7b).

Among the similar features in the group, medium-wise temporal trends of $\left\langle\Delta\left[\mathrm{HbO}_{2}\right]>,\langle\Delta[\mathrm{HHb}]>\right.$ and $\langle\Delta[\mathrm{oxCCO}]\rangle$ across all the subject mice, the following are worth mentioning: (1) both the haemodynamic and the metabolic responses present comparable and close magnitudes in the vascular and the grey matter media, respectively, for all the experimental phases; (2) almost equivalent timings in the temporal dynamics of both the haemodynamic and the metabolic responses in the vascular and the grey matter, respectively; (3) comparable variance and influence of noise in the values of the spatial average changes in the concentrations of $\mathrm{HbO}_{2}, \mathrm{HHb}$ and oxCCO over the entire FOV, as inferred from the comparison of the standard deviations of the temporal trends of $\left\langle\Delta\left[\mathrm{HbO}_{2}\right]\right\rangle,\langle\Delta[\mathrm{HHb}]\rangle$ and $<\Delta$ [oxCCO] $>$; (4) a delay in the inception and peaking of the metabolic responses, compared to haemodynamic ones; (5) the occurrence of an initial dip of similar magnitude in the haemodynamic responses of $\mathrm{HbO}_{2}$ in the pial vasculature medium during the first time window of hyperoxia; (6) the manifestation of a quasi-stationary plateau in the values of $\langle\Delta[\mathrm{HHb}]>$ in the pial vasculature during the last time windows of anoxia.

\section{CONCLUSION}

We presented a novel HSI system, called hNIR, capable of simultaneously monitoring and quantifying the haemodynamic and metabolic states of the exposed cerebral cortex in vivo, via mapping of the relative changes in the concentrations of the two forms of haemoglobin $\left(\mathrm{HbO}_{2}\right.$ and $\left.\mathrm{HHb}\right)$ and the oxidative state of $\mathrm{CCO}$ (oxCCO). In future studies, a quantitative assessment of the depth sensitivity of the hNIR system could also be performed, in order to provide a full characterisation of the in vivo performances of the setup.

The application of HSI to the simultaneous mapping and quantification of the haemodynamic and metabolic states of the exposed cortex via targeting of haemoglobin and $\mathrm{CCO}$ was an area that had not been previously explored in literature before this (to the best of our knowledge) and the results of this work could open the field to several potential investigations on a number of conditions and scenarios involving the inner working of the brain. These could include life science and neurological research on the physiological responses and mechanisms in animal studies of models of multiple sclerosis (MS) and of hypoxic-ischaemic injuries, such as stroke and dementia. The hNIR system is also compact enough to be used within an operation room during neurosurgery, allowing HSI of the human exposed cortex during tumour resection or during epilepsy surgery. Furthermore, hNIR could be modified and optimised in the future to increase temporal resolution to sub-second scale, enabling investigations of brain functional activation and neurovascular coupling.

Finally, the hNIR system and the associated methodologies are versatile enough so that they could also be tailored for multiple biomedical applications outside of the study of the brain and explored in future work.

\section{REFERENCES}

[1] G. Lu and B. Fei, "Medical hyperspectral imaging: a review," $J$. Biomed. Opt., vol. 19, no. 1, p. 010901, Jan. 2014.

[2] H. Akbari and Y. Kosugi, "Hyperspectral imaging: A new modality in surgery," Recent Adv. Biomed. Eng., pp. 223-240, Oct. 2009.

[3] M. A. Calin, S. V. Parasca, D. Savastru, and D. Manea, "Hyperspectral imaging in the medical field: Present and future," Appl. Spectrosc. Rev., vol. 49, no. 6, pp. 435-447, Aug. 2014.

[4] R. Lucas, A. Rowlands, O. Niemann, and R. Merton, "Hyperspectral Sensors and Applications," in Advanced Image Processing Techniques for Remotely Sensed Hyperspectral Data, Berlin, Heidelberg: Springer Berlin Heidelberg, 2004, pp. 11-49.

[5] L. Giannoni, F. Lange, and I. Tachtsidis, "Hyperspectral imaging solutions for brain tissue metabolic and hemodynamic monitoring: past, current and future developments," J. Opt., vol. 20, no. 4, p. 044009, Mar. 2018.

[6] F. Scholkmann et al., "A review on continuous wave functional nearinfrared spectroscopy and imaging instrumentation and methodology," Neuroimage, vol. 85, pp. 6-27, 2014.

[7] E. M. C. Hillman, "Optical brain imaging in vivo: techniques and applications from animal to man.," J. Biomed. Opt., vol. 12, no. 5, p. $051402,2007$.

[8] Y. Ma et al., "Wide-field optical mapping of neural activity and brain haemodynamics: considerations and novel approaches.," Philos. Trans. R. Soc. Lond. B. Biol. Sci., vol. 371, no. 1705, p. 20150360, Oct. 2016.

[9] G. Bale, C. E. Elwell, and I. Tachtsidis, "From Jöbsis to the present day: a review of clinical near-infrared spectroscopy measurements of cerebral cytochrome-c-oxidase," J. Biomed. Opt., vol. 21, no. 9, p. 091307, 2016.

[10] M. G. Mason, P. Nicholls, and C. E. Cooper, "Re-evaluation of the near infrared spectra of mitochondrial cytochrome c oxidase: Implications for non invasive in vivo monitoring of tissues," Biochim. Biophys. Acta - Bioenerg., vol. 1837, no. 11, pp. 1882-1891, Nov. 2014.

[11] G. Bale, S. Mitra, J. Meek, N. Robertson, and I. Tachtsidis, "In-Vivo Measurements of Cerebral Changes in Cytochrome-c-Oxidase using Broadband Near-Infrared Spectroscopy in Perinatal Hypoxic-Ischaemic Encephalopathy," in Biomedical Optics 2014, 2014, p. BS3A.39.

[12] S. Brigadoi et al., "Image reconstruction of oxidized cerebral cytochrome $\mathrm{C}$ oxidase changes from broadband near-infrared 
spectroscopy data," Neurophotonics, vol. 4, no. 2, p. 021105, Apr. 2017.

[13] C. Kolyva, A. Ghosh, I. Tachtsidis, D. Highton, M. Smith, and C. E. Elwell, "Dependence on NIRS source-detector spacing of cytochrome c oxidase response to hypoxia and hypercapnia in the adult brain," in Adv. Exp. Med. Biol., 2013, vol. 789, pp. 353-359.

[14] L. Giannoni, F. Lange, and I. Tachtsidis, "Investigation of the quantification of hemoglobin and cytochrome-c-oxidase in the exposed cortex with near-infrared hyperspectral imaging: a simulation study," $J$. Biomed. Opt., vol. 25, no. 04, p. 1, Apr. 2020.

[15] Y. Yu and S. T. Acton, "Speckle reducing anisotropic diffusion," IEEE Trans. Image Process., vol. 11, no. 11, pp. 1260-1270, Nov. 2002.

[16] V. S. Frost, J. A. Stiles, K. S. Shanmugan, and J. C. Holtzman, "A Model for Radar Images and Its Application to Adaptive Digital Filtering of Multiplicative Noise," IEEE Trans. Pattern Anal. Mach. Intell., vol. PAMI-4, no. 2, pp. 157-166, Mar. 1982.

[17] D. T. Delpy, M. Cope, P. van der Zee, S. Arridge, S. Wray, and J. Wyatt, "Estimation of optical pathlength through tissue from direct time of flight measurement.," Phys. Med. Biol., vol. 33, no. 12, pp. $1433-1442,1988$.

[18] S. J. Matcher, M. Cope, and D. T. Delpy, "Use of the water absorption spectrum to quantify tissue chromophore concentration changes in near-infrared spectroscopy.," Phys. Med. Biol., vol. 39, no. 1, pp. 17796, Jan. 1994.

[19] A. Ben-Israel and T. N. E. Greville, Generalized Inverses: Theory and Applications. Springer, 2000.

[20] S. Kleiser, N. Nasseri, B. Andresen, G. Greisen, and M. Wolf, "Comparison of tissue oximeters on a liquid phantom with adjustable optical properties," Biomed. Opt. Express, vol. 7, no. 8, p. 2973, Aug. 2016.

[21] S. Hyttel-Sorensen, S. Kleiser, M. Wolf, and G. Greisen, "Calibration of a prototype NIRS oximeter against two commercial devices on a blood-lipid phantom," Biomed. Opt. Express, vol. 4, no. 9, p. 1662, Sep. 2013.

[22] F. Lange, L. Dunne, L. Hale, and I. Tachtsidis, "MAESTROS: A Multiwavelength Time-Domain NIRS System to Monitor Changes in Oxygenation and Oxidation State of Cytochrome-C-Oxidase," IEEE J. Sel. Top. Quantum Electron., vol. 25, no. 1, pp. 1-12, Jan. 2019.

[23] F. Martelli and G. Zaccanti, "Calibration of scattering and absorption properties of a liquid diffusive medium at NIR wavelengths CW method," Opt. Express, vol. 15, no. 2, p. 486, Jan. 2007.

[24] P. Di Ninni, F. Martelli, and G. Zaccanti, "Effect of dependent scattering on the optical properties of Intralipid tissue phantoms," Biomed. Opt. Express, vol. 2, no. 8, p. 2265, Aug. 2011.

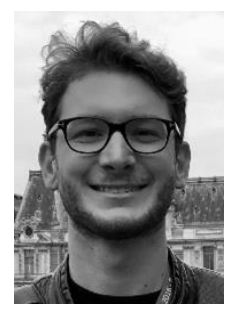

Luca Giannoni received the B.Sc. degree in nuclear engineering in 2013 and the M.Sc. (Hons) degree in nuclear engineering in 2016, both from Università di Pisa, Italy. From 2015 to 2016 he was a visiting research student at the Department of Nuclear, Plasma and Radiological Engineering at University of Illinois at Urbana-Champaign (UIUC), U.S.A, working on X-ray fluorescence and luminescence emission tomography. Since 2016, he has been a Ph.D. student in medical imaging and a Marie Curie Early Stage Researcher at the Biomedical Optics Research Laboratory, Department of Medical Physics and Biomedical Engineering, University College London (UCL), U.K. His current work focuses on developing a hyperspectral imaging benchtop system to monitor and study the haemodynamic and metabolic states of the exposed cerebral cortex in small animals, in particular related to traumatic brain injuries and cerebral oxygen deficiency conditions.

Frédéric Lange received his M.Sc. degree in neuroscience and healthcare imaging from the University of Caen Basse-Normandie, Caen, France, in 2012, and his Ph.D. degree in biomedical optics from the University of Lyon and INSA de LYON, Lyon, France, in 2016. Since 2016, he has been a Research Associate with the Biomedical Optics Research Laboratory, Department of Medical Physics and Biomedical Engineering, University College London, London, U.K. His main current research interests are in the development of diffuse optics instrumentation and methodologies (time-resolved diffuse spectroscopy, broadband near-infrared spectroscopy, diffuse correlation spectroscopy) for biomedical applications.

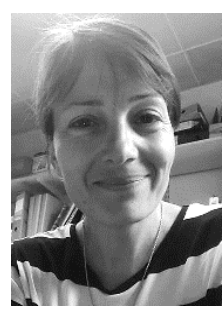

Marija Sajic received the M.D. from the Medical School, University of Belgrade, Serbia in 2002, and the $\mathrm{Ph} . D$. from King's College London, in 2007. Since 2008 she has been a Research Associate, and since 2016 a Senior Research Associate at the Department of Neuroinflammation, UCL Institute of Neurology. Her focus is on in vivo study of mitochondria in physiological and pathological conditions. She is the recipient of the P.K. Thomas award from the PNS Society in 2012, for her work on elucidating relationship between impulse conduction and mitochondrial transport along myelinated and unmyelinated axons, in vivo.

Kenneth J. Smith received his B.Sc. in physiology from the University of London, and then his Ph.D. at the Institute of Neurology working with Ian McDonald on the conduction properties of remyelinated axons. After a post-doc at the Institute, with Hugh Bostock looking at conduction along demyelinated axons, he spent five years in Chicago examining the biophysical properties of the node of Ranvier. After a further five years in Virginia, he returned to the Guy's Hospital Campus and King's College London, working with Richard Hughes. In 2007 he moved back to the Institute of Neurology and became Head of the Department of Neuroinflammation. His research examines the pathophysiological properties of the inflamed brain, studied by electrophysiology, confocal imaging, and light and electron microscopy. His research has led directly to the use of sodium channel blocking agents in neuroprotective clinical trials. In recent years he has particularly explored how inflammation can deprive the brain of sufficient oxygen, and thus of sufficient energy, resulting in loss of function, demyelination, degeneration and atrophy. Most recently his studies have indicated the value of strategies to maintain tissue oxygenation as a therapy to protect mitochondrial and neurological function, and tissue integrity in neuroinflammatory diseases such as multiple sclerosis.

Ilias Tachtsidis received the B.Eng. (Hons) degree in medical engineering from Bournemouth University, U.K., in 2000, the M.Sc. degree in medical electronics and medical physics from the Queen Mary University, London, U.K., in 2001, and a Ph.D. degree in medical physics and biomedical optics from University College London, UK, in 2005. He is Wellcome Trust Senior Fellow, the Head of the Multi-Modal Spectroscopy Group, Department of Medical Physics and Biomedical Engineering, and a Reader in biomedical engineering with the University College London, London, U.K. His research interests are multidisciplinary, crossing the boundaries between engineering, physics, neuroscience, and clinical medicine. The technical focus of his work is the development and use of non-invasive optical instruments and techniques for monitoring brain oxygenation, haemodynamics, and metabolism. The principal challenge of his research is the non-invasive measurement, with near-infrared spectroscopy, of cytochrome-c-oxidase, a mitochondrial enzyme responsible for cellular energy production. Dr Tachtsidis is a recipient of the Melvin H. Knisely Award, in 2007, from the International Society of Oxygen Transport to Tissue, acknowledging a Young Investigator for outstanding achievements in research related to oxygen transport to tissue. 